\title{
GSK-3 $\beta$ inhibitor 6-bromo-indirubin-3'-oxime promotes both adhesive activity and drug resistance in colorectal cancer cells
}

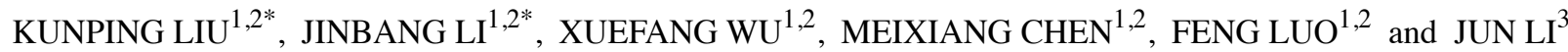 \\ ${ }^{1}$ Department of Pathology, The Sixth Affiliated Hospital of Guangzhou Medical University, Qingyuan \\ People's Hospital, Qingyuan, Guangdong 511518; ${ }^{2}$ Department of Pathology, Qingyuan People's Hospital, \\ Jinan University, Qingyuan, Guangdong 511518; ${ }^{3}$ Department of General Surgery, The First Affiliated \\ Hospital of Guangzhou Medical University, Guangzhou, Guangdong 510120, P.R. China
}

Received May 27, 2017; Accepted October 2, 2017

DOI: $10.3892 /$ ijo.2017.4163

\begin{abstract}
Multi-targets inhibitor 6-bromo-indirubin-3'-oxime (BIO) has diverse biological effects on cancer cells. The key component of the $\beta$-catenin destruction complex glycogen

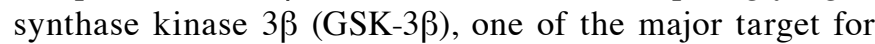
$\mathrm{BIO}$, polyubiquitination and degradation of the main oncoprotein $\beta$-catenin in colorectal cancer (CRC). In the present study, we evaluated the effect of BIO on drug resistance and biological properties of CRC cells. Whole-genome transcriptional profiling revealed that differentially expressed genes were mainly centered on well-characterized signaling pathways including stem cell, cell adhesion and cell growth in BIO-treated CRC cells. BIO treatment downregulated migration and invasion abilities of CRC cells, accompanying with MMP-9 downregulated and E-cadherin upregulated CRC cells. BIO treatment decreased apoptosis induced by 5-Fu/DDP in CRC SW480 cells. In addition, BIO treatment reversed the 5-Fu-induced $\mathrm{CD} 133^{+}$cell downregulation trend in CRC SW620 cells. After incubation with BIO, the expression levels of EpCAM, TERT and DCAMKL-1 proteins were upregulated in CRC cells. BIO treatment downregulated the activity of GSK-3 $\beta$, upregulated and transported $\beta$-catenin to the nucleus in CRC cells. Our findings reveal that BIO treatment upregulated stemness, adhesive and chemoresistance of
\end{abstract}

Correspondence to: Dr Kunping Liu, Department of Pathology, The Sixth Affiliated Hospital of Guangzhou Medical University, Qingyuan People's Hospital, Qingyuan, Guangdong 511518, P.R. China

E-mail: gdqylkp@163.com

Dr Jun Li, Department of General Surgery, The First Affiliated Hospital of Guangzhou Medical University, Guangzhou, Guangdong 510120, P.R. China

E-mail: jun-li621120@163.com

${ }^{*}$ Contributed equally

Key words: colorectal cancer, GSK-3 $\beta$, drug resistance, 6-bromoindirubin-3'-oxime, WNT signaling pathway
CRC cells. GSK-3 $\beta$ inhibition and WNT/ $\beta$-catenin activation by $\mathrm{BIO}$, may partly result in the biological behavior alterations in CRC cells.

\section{Introduction}

Colorectal cancer (CRC) is a common malignant tumor, 5-fluorouracil (5-Fu) and cisplatin (DDP) are commonly used in the chemotherapy of CRC, but the resistance to chemotherapy is one of the main obstacles in CRC treatment, which leads to the failure of chemotherapy $(1,2)$. WNT signaling pathway effector $\beta$-catenin is the main oncoprotein in CRC subtypes CMS2 (consensus molecular subtypes 2) (3). Numerous studies indicate that the drug resistance abilities enhancing, angiogenesis, presence of highly resistant cancer stem cells (CSCs), dysregulation of cell cycle and apoptosis in CRC cells were mainly due to $\mathrm{WNT} / \beta$-catenin overactivation (4-7).

Genetic mutations in the $\beta$-catenin destruction complex, which consists of Apc, Axin, Ck1 and GSK-3 $\beta$, led to $\beta$-catenin transportation to the nucleus and over activation in most CRC cases (8-10). These genes may be the potential anti-multidrug resistance targets for $\mathrm{CRC}$, for their demonstrable effect on $\beta$-catenin polyubiquitination and degradation $(11,12)$. The serine/threonine kinase GSK-3 was initially identified in rat skeletal muscle as a kinase that phosphorylated and inactivated glycogen synthase (GS) $(13,14)$. The two known GSK-3 family members, GSK-3 $\alpha$ and GSK-3 $\beta$, were ubiquitously expressed and highly conserved. GSK-3 $\beta$ activity is regulated by phosphorylation at serine 9, threonine 216 and other residues. GSK-3 $\beta$ regulates a variety of physiological functions, including cell proliferation, differentiation, motility, cycle progression, apoptosis, and has become an attractive target for chemotherapy (15-18). Therefore, numerous studies focus on the biological behavior alteration of CRC cells after activation/ inhibition of GSK-3 $\beta$ by small molecule compounds.

Small-molecule ATP competitive BIO is regarded as a classical GSK-3 inhibitor $(19,20)$. It inhibits not only GSK-3 $\beta$, but also GSK-3 $\alpha$. More recent research suggest that BIO inhibits also JAK1 and TYK2 (21). Importantly, the role of $\mathrm{BIO}$ in the biological properties of cells, which joins two or more metabolic and signal transduction pathways, may vary between cell types. Studies reported that BIO suppressed the 
proliferation of ovarian cancer cells (22) and induces apoptosis of human melanoma cells (21), but enhanced the proliferation capacity of rat marrow-derived mesenchymal stem cells and cardiomyocytes $(23,24)$.

In the present study, we evaluated the effects of BIO on stemness, adhesive and drug resistance activities of CRC SW480 and SW620 cells. In addition, the effects of BIO on GSK-3 $\beta$ phosphorylation and WNT/ $\beta$-catenin activity were studied. Our results indicated that BIO affects stemness, adhesive and chemoresistance abilities of CRC cells. The biological behavior alteration in $\mathrm{BIO}$ treated CRC cells may partly attribute to $\mathrm{WNT} / \beta$-catenin activation.

\section{Materials and methods}

Chemicals. BIO was purchased from Sigma-Aldrich (St. Louis, MO, USA) and 5-fluorouracil (5-Fu) was from Shanghai Xudong Haipu Pharmaceutical Co., Ltd. (Shanghai, China). Cisplatin (DDP) was purchased from Yunnan Biovalley Dengzhanhua Pharmaceutical Co., Ltd. (Kunming, China).

Cell culture. Human colon adenocarcinoma SW480 and SW620 cells were grown as monolayers and routinely cultured as previously described (12). Cell lines were subjected to DNA tests and authenticated in our previous studies (12). The cells were cultured in RPMI-1640 medium (Gibco, Grand Island, NY, USA) with $10 \%$ fetal bovine serum [FBS; Tianjin Institute of Blood (TBD), Tianjin, China] at $37^{\circ} \mathrm{C}$ under an atmosphere of $5 \% \mathrm{CO}_{2}$. Both cell lines are regularly authenticated on the basis of viability, recovery, growth, morphology and chemical response as well, were most recently confirmed 3-6 months before use by using a short tandem repeat method.

Microarray analysis. The main observation target after BIO treatment is the expression level of WNT signaling pathway effector $\beta$-catenin. The CRC SW480 and SW620 cells were treated for $24 \mathrm{~h}$ with $4 \mu \mathrm{M}$ BIO in our experiments. Total RNA (3 repeated) was extracted from CRC SW480 and SW620 cells. Integrity and concentration of RNA was assessed after RNA extraction and prior to sample labeling. NimbleGen One-Color DNA Labeling kit was used for sample labeling. Hybridization was performed in NimbleGen Hybridization System (Roche). After washing, slides were scanned with Axon GenePix 4000B scanner. Data were extracted and normalized using NimbleScan v2.5 software. Results are provided in the NimbleScan Generated Data Folder. Further data analysis was performed using Agilent GeneSpring GX v11.5 software and the GenCLiP 2.0 online tool (http://ci.smu.edu.cn/).

Apoptosis analysis. The cells were treated for $24 \mathrm{~h}$ with BIO (4 $\mu \mathrm{mol} / \mathrm{l}), 5-\mathrm{Fu}(50 \mu \mathrm{mol} / \mathrm{l})$, DDP $(8 \mu \mathrm{mol} / \mathrm{l}), \mathrm{BIO}(4 \mu \mathrm{mol} / \mathrm{l})+$ DDP $(8 \mu \mathrm{mol} / \mathrm{l})$ and BIO $(4 \mu \mathrm{mol} / \mathrm{l})+5$-Fu $(50 \mu \mathrm{mol} / \mathrm{l})$, separately. The cells were measured using FACSAria flow cytometer (BD Biosciences, San Jose, CA, USA), and Annexin V (+) cells were counted for apoptotic cells after Annexin V/fluorescein isothiocyanate/propidium iodide (FITC/PI) (BD Biosciences) double staining. The experiment was performed in triplicate.

Cell cycle analysis. The cells were treated for $24 \mathrm{~h}$ with BIO ( $4 \mu \mathrm{mol} / \mathrm{l})$. The cells were harvested and fixed in $70 \%$ ice-cold ethanol at $4^{\circ} \mathrm{C}$ for $30 \mathrm{~min}$. After washed with phosphate-buffered saline (PBS), the cells were incubated with propidium iodide staining buffer (BD Biosciences) for $15 \mathrm{~min}$ and analyzed by flow cytometer. The experiment was performed in triplicate.

Flow cytometric analysis of CD133-positive cell population. Human colon adenocarcinoma SW480 and SW620 cell lines $\left(1 \times 10^{6}\right)$ were detached by treatment with $0.25 \%$ trypsin/EDTA and washed twice with PBS. The cells were then resuspended in $100 \mu \mathrm{l}$ of staining buffer containing 1\% FBS and place on ice for 20 min to block Fc receptors. After incubating with primary phycoerythrin antihuman CD133 antibody (Miltenyi Biotec GmbH, Bergisch Gladbach, Germany) for another $10 \mathrm{~min}$ on ice in the dark, the cells were washed twice with $1 \mathrm{ml}$ of ice-cold staining buffer and centrifuged $(300 \mathrm{x} \mathrm{g})$ for $10 \mathrm{~min}$ at $4^{\circ} \mathrm{C}$. Cells resuspended in $0.3 \mathrm{ml}$ of $2 \%$ formaldehyde fixation buffer were analyzed using a FACSAria flow cytometer and CellQuest software (BD Biosciences). All flow cytometric results were obtained from two independent experiments performed in triplicate.

Transwell migration and boyden chamber invasion assays. For the Transwell migration assay, $10^{5}$ cells in $100 \mathrm{ml}$ of serum-free DMEM media were seeded in triplicate in each fibronectin-coated polycarbonate membrane insert in a Transwell apparatus (Corning, Inc., Corning, NY, USA). A total of $600 \mu \mathrm{l}$ of $10 \%$ NCS in DMEM was added to the bottom chamber. SW480 and SW620 cells were incubated at $37^{\circ} \mathrm{C}$ for $12 \mathrm{~h}$. The inserts were washed twice with prewarmed PBS. Cells adhered on the lower surface were fixed with $100 \%$ methanol at RT for $15 \mathrm{~min}$ and stained with hematoxylin for $15 \mathrm{~min}$. Cell numbers in six predetermined fields in each replicate were counted under the microscope (Nikon Eclipse 80i system; x200). All assays were independently repeated at least three times. Cell invasion assays were performed as the migration assay except the Transwell membrane was precoated with $24 \mathrm{mg} / \mathrm{ml}^{-1}$ Matrigel (R\&D Systems, Minneapolis, MN, USA) and the cells were incubated for 24 and $18 \mathrm{~h}$, respectively.

Western blot analysis. Following treatment with different drugs for $24 \mathrm{~h}$, the cells were collected and lysed. Protein content was measured by the BCA protein assay kit (Beyotime Institute of Biotechnology, Shanghai, China) and $20 \mu \mathrm{g}$ proteins per lane was separated by $8-12 \%$ sodium dodecyl sulfate polyacrylamide gel electrophoresis (SDS-PAGE) and transferred onto nitrocellulose membranes. Specific protein bands were achieved with an ECL detection reagent (Pierce, Rockford, IL, USA). Anti- $\beta$-catenin (Cell Signaling Technology, Danvers, MA, USA) dilutions were 1:1,000. AntiDCAMKL-1 and anti-TERT (Abcam, Cambridge, MA, USA) dilutions were 1:300 and 1:800. Anti-EpCAM, anti-GAPDH and anti- $\alpha$-tubulin (Cell Signaling Technology) dilutions were 1:500, 1:1,000 and 1:1,000, respectively. Anti-CD44v6 (Cell Signaling Technology) dilution was 1:1,000. Horseradish peroxidase (HRP)-conjugated goat anti-rabbit and goat antimouse IgG antibodies (ProteinTech Group, Chicago, IL, USA) dilutions were 1:3,000. $\alpha$-tubulin was used as a protein loading control. The images were captured with ChemiDoc ${ }^{\mathrm{TM}}$ CRS+ Molecular Imager (Bio-Rad Laboratories, Hercules, 


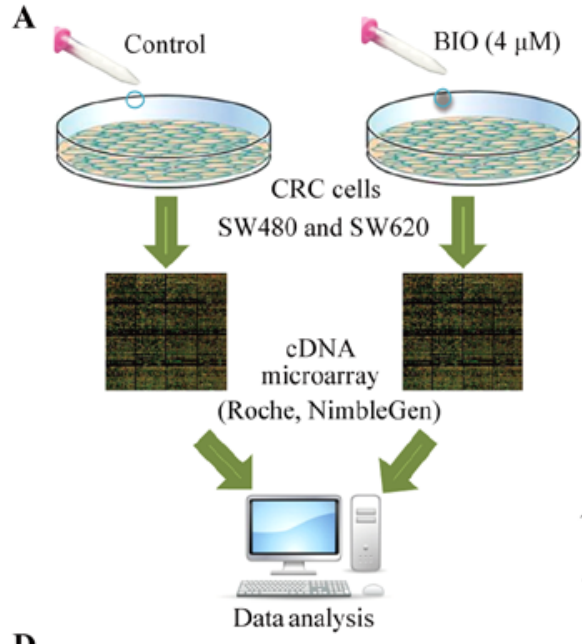

D

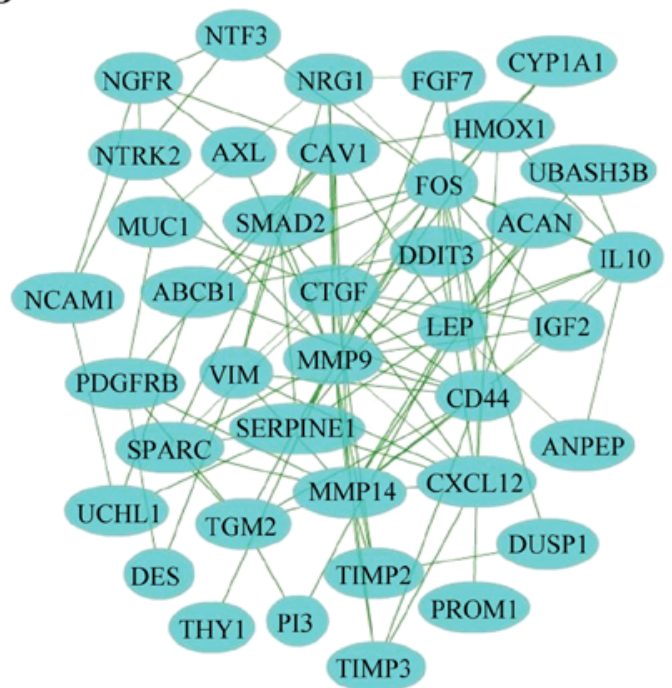

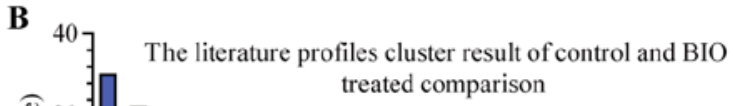

B 407 The literature profiles cluster result of control and BIO treated comparison

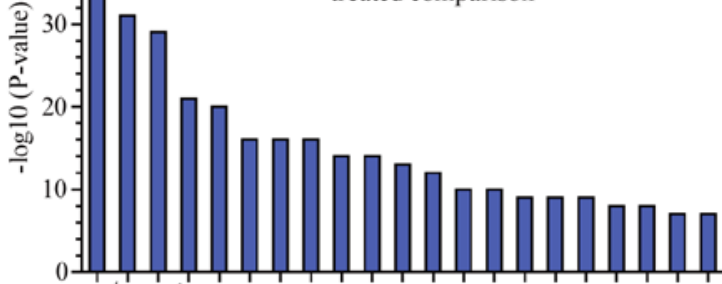

WNK2

DUSP6

UCHL1

AXL

TGM2

MUC1

TIMP3

DDIT3

CYP1Al

MMP14

FGF7

HMOXI

ABCB 1

IGF2

LEP

FOS

CD44

SMAD2

IL10

MMP9
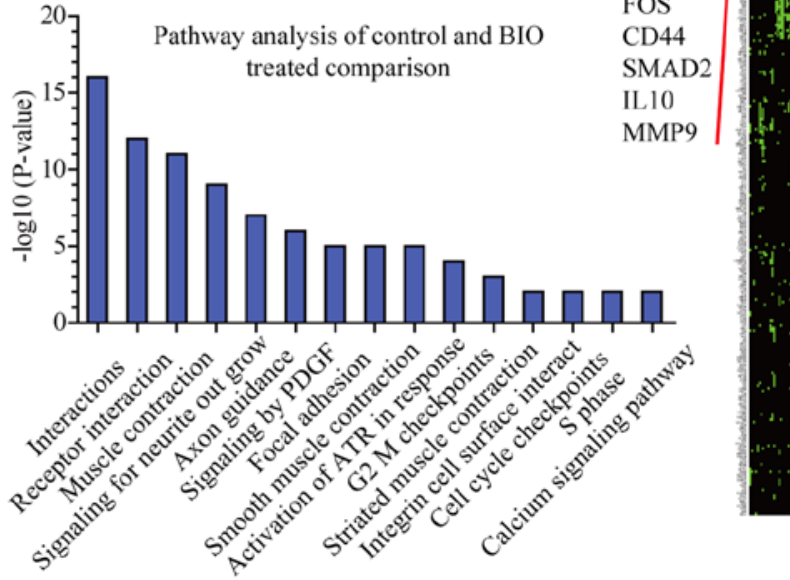

\begin{abstract}
Figure 1. Functional annotations of the differentially expressed genes before and after treatment of SW480 and SW620 cells with BIO based on GenCLiP 2.0 online tool analysis. (A) The model for microarray-based analysis of differentially expressed genes before and after treatment with SW480 and SW620 cells with BIO $(4 \mu \mathrm{M})$ for $24 \mathrm{~h}$. (B) The literature profile-based keywords cluster result of control and BIO-treated comparison. (C) Heatmap shows the clustering result generated from differentially expressed genes and literature profile-based keywords based on GenCLiP 2.0 online tool analysis before and after BIO treatment in SW480 and SW620 cells. Each row and column in the heat map representation is differentially expressed genes and literature profile-based keywords, respectively. (D) Gene-gene interaction network of control and BIO treated comparison generated using the GenCLiP 2.0 online tool. (E) Pathway
\end{abstract} analysis of control and BIO-treated comparison.

CA, USA). The density of the protein band was quantitated using the Quantity One software (Bio-Rad Laboratories). The experiment was performed in triplicate.

Statistical analysis. Statistical analysis was performed with the SPSS 13.0 software package (SPSS, Inc., Chicago, IL, USA). Two-class significance analysis of microarrays (SAM) was used to identify genes that were differentially expressed in before and after BIO-treated CRC SW480 and SW620 cells, the statistical significance was assessed by a false discovery rate (FDR). Data are presented as mean $\pm \mathrm{SD}$. One way analysis of variance (ANOVA) was used for apoptosis, CD133, cell cycle and western blot data analyses. $\mathrm{P}<0.05$ was considered to indicate a statistically significant difference.

\section{Results}

Screening differentially expressed genes before and after $B I O$ treatment in SW480 and SW620 cells. In order to survey the biological behavior alteration induced by BIO in CRC cells, we conducted cDNA microarrays (Roche Nimble Gen) to pick out the differentially expressed genes before and after BIO-treated in CRC cells (Fig. 1A). CRC cell lines SW480 and SW620 were submitted to the gene chip experiment in the present study. The SAM supervised analysis identified differentially expressed genes of SW480 and SW620 cells after treatment with BIO, respectively. The 545 differentially expressed genes consistently upregulated or downregulated in SW480 and SW620 cells after treatment with BIO were selected and used for further functional annotation and enrichment analysis. As shown in Fig. 1B, functional annotations of a total 545 differentially expressed genes, which were jointly upregulated or downregulated in SW480 and SW620 cells after treatment with $\mathrm{BIO}$, were generated using the GenCLiP 2.0 online tool. As shown in Fig. 1C, heatmap showing the clustering result of differentially expressed genes and literature profile keywords based on GenCLiP 2.0 online tool analyzed between before and after BIO-treated in SW480 and SW620 cells. 
A

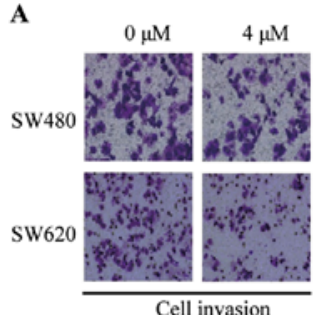

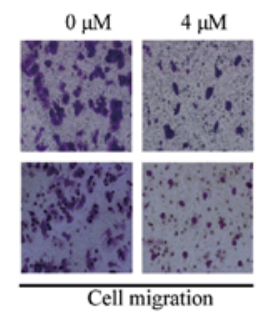

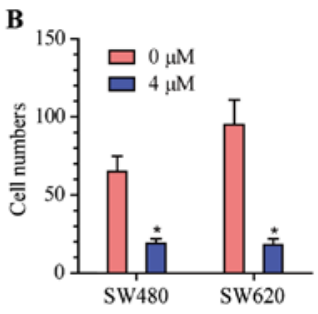

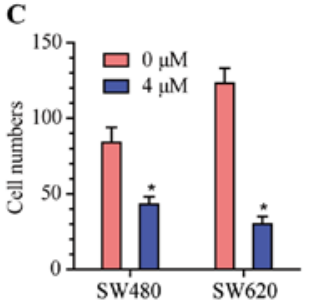
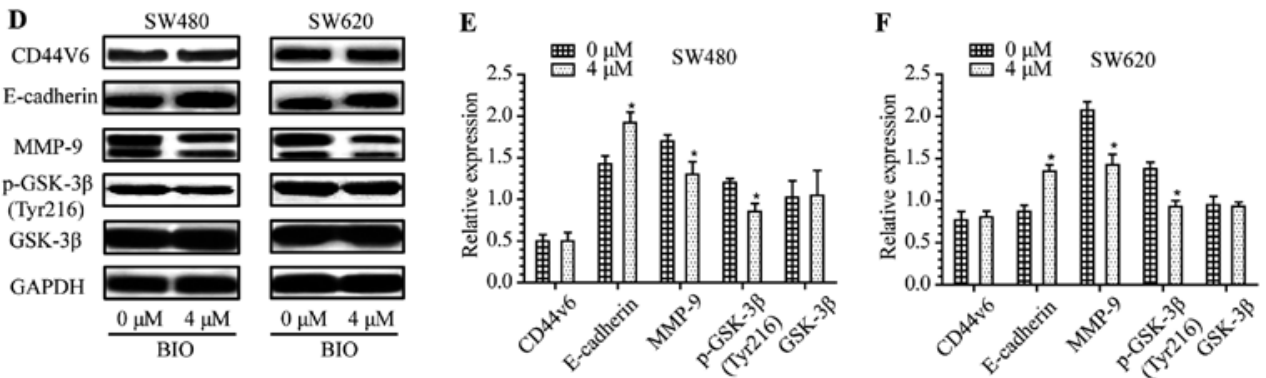

Figure 2. Comparison of migration/invasion abilities and related proteins expression of SW480 and SW620 cells after treatment with BIO. (A-C) Transwell assays and boyden chamber assays evaluated the migration and invasion abilities alteration of SW480 and SW620 cells after treatment with BIO (4 $\mu \mathrm{M})$ for

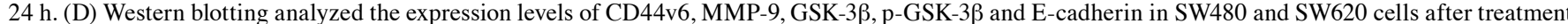
with BIO $(4 \mu \mathrm{M})$ for $24 \mathrm{~h}$. (E and F) Values are expressed as protein/GAPDH. The density of the protein band was quantitated using Quantity One software. The data are expressed as mean \pm SD of three experiments. ${ }^{*} \mathrm{P}<0.05$ compared with the control $(0 \mu \mathrm{M})$ group.

A
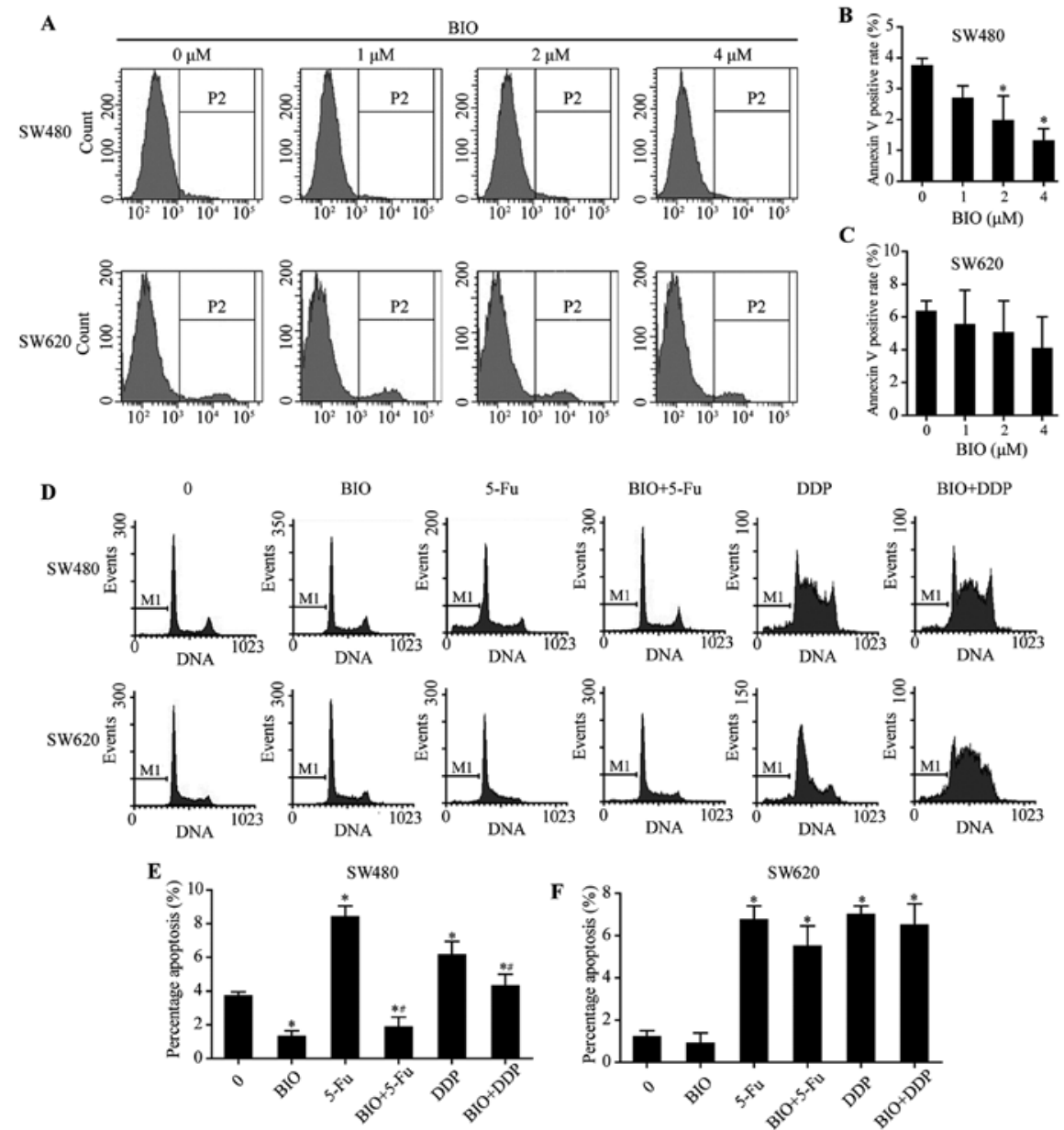

Figure 3. The effects of BIO on apoptosis induced by 5-FU/DDP of SW480 and SW620 cells. (A) Flow cytometric analysis of Annexin V-positive cells in SW480 and SW620 cells after treatment with different concentrations of BIO (0,1,2 and $4 \mu \mathrm{M})$. (B and C) The rate of Annexin V-positive cells in SW480 and SW620 cells after treatment. (D) Flow cytometric analysis of Annexin V-positive cells in SW480 and SW620 cells either incubated with 5-Fu/DDP alone or combining with BIO $(4 \mu \mathrm{M})$ for $24 \mathrm{~h}$. (E and F) The rate of Annexin V-positive cells in SW480 and SW620 cells after treatment. All Annexin V-positive cells were considered apoptotic cells, and their percentage was calculated among the total number of cells. Each bar represents the means \pm SD of three experiments. ${ }^{*} \mathrm{P}<0.05$ compared with the control. ${ }^{\#} \mathrm{P}<0.05$ compared with 5-Fu-treated or DDP-treated cells. 
A
SW480
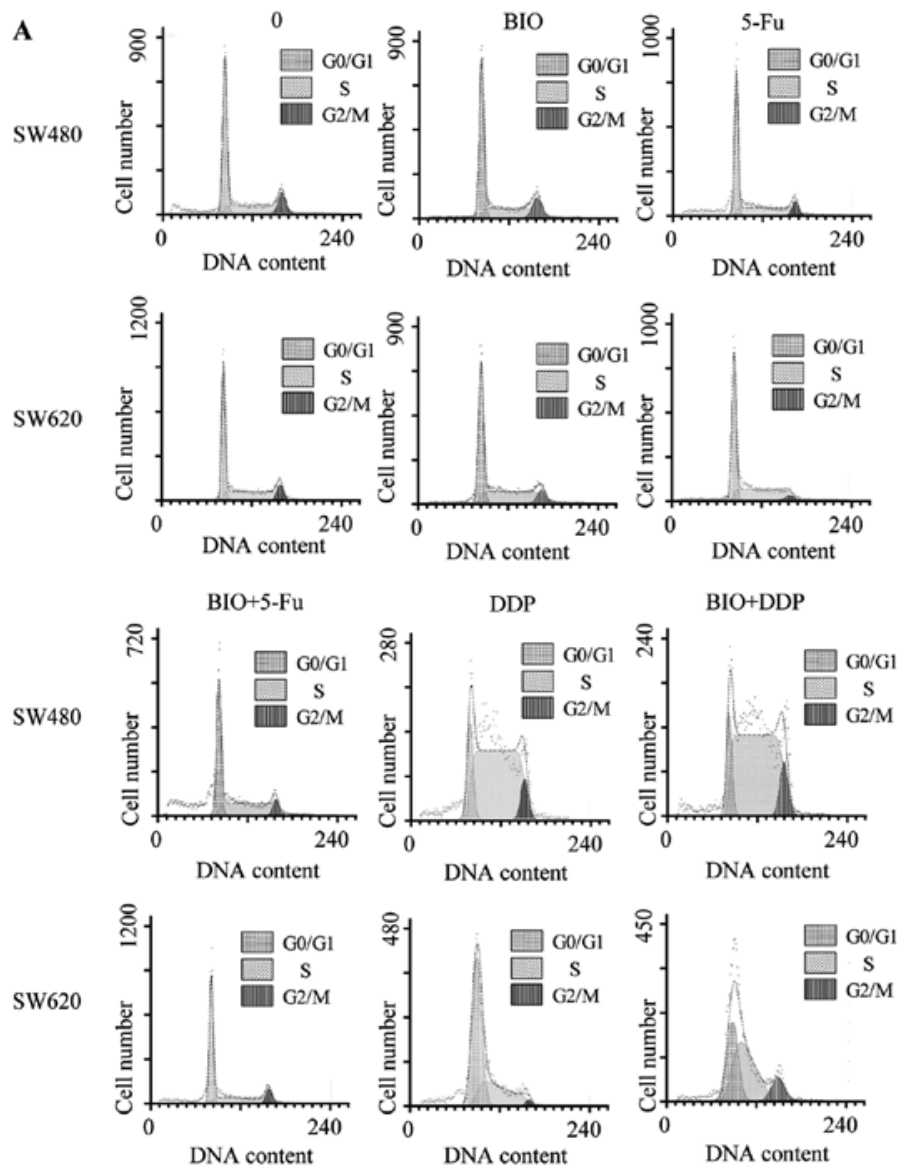
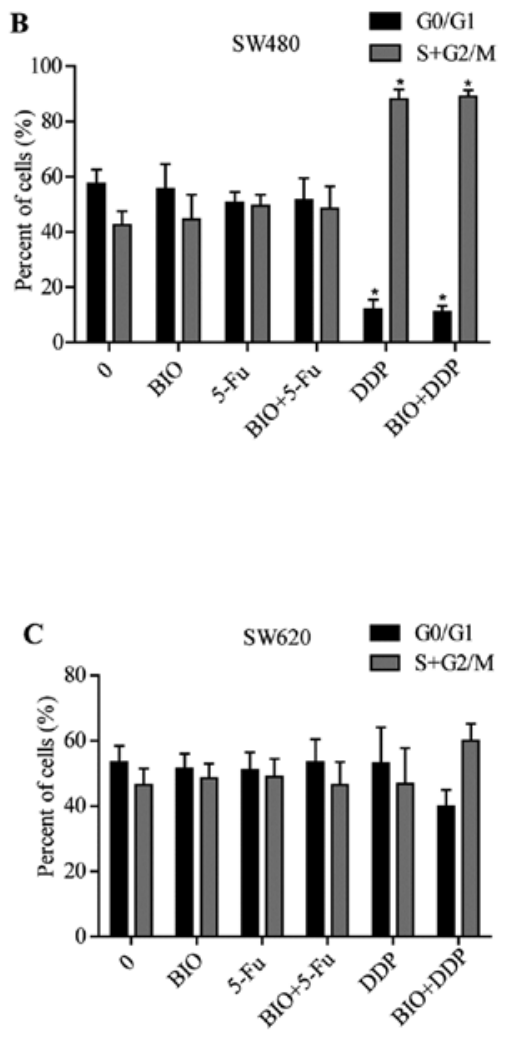

Figure 4. The effects of BIO on cell cycle distribution of SW480 and SW620 cells. (A) Flow cytometric analysis of cell cycle distribution in SW480 and SW620 cells either incubated with 5-Fu/DDP alone or combining with BIO $(4 \mu \mathrm{M})$ for $24 \mathrm{~h}$. (B and C) The cell cycle distribution in SW480 cells and SW620 cells after treatment with 5-Fu/DDP alone or combined with $\mathrm{BIO}(4 \mu \mathrm{M})$ for $24 \mathrm{~h}$. Results are presented as means $\pm \mathrm{SD}$ of three experiments. "P<0.05 compared with the control.

The key literature profile-based keywords before and after BIO-treatment in SW480 and SW620 cells are extracellular matrix, basement membrane, transforming growth factor, endothelial growth factor, mesenchymal stem cell, cell differentiation, cell adhesion and cell migration. Gene-gene interaction network of 41 selected genes were generated and shown in Fig. 1D using GenCLiP 2.0 online tool. The differential signaling pathway comparisons between before and after BIO-treatment are shown in Fig. 1E.

Effect of BIO on cell migration, invasion and GSK-3 $\beta$ activity in SW480 and SW620 cells. Functional annotations of the differentially expressed genes indicated that cell migration and invasion related genes were changed significantly after treatment with BIO in SW480 and SW620 cells. The migration and invasion abilities were determined through Transwell assays and boyden chamber assays (Fig. 2A). As shown in Fig. 2B and C, the migration and invasion abilities of SW480 and SW620 cells were significantly decreased after treatment with $\mathrm{BIO}(\mathrm{P}<0.05)$.

Detection the key components of cell migration and invasion relative proteins by western blotting demonstrated that the expression level of MMP-9 was decreased, while the level of E-cadherin was elevated after BIO treatment in SW480 and SW620 cells (Fig. 2D-F; P<0.05), but the expression level of CD44V6 was not altered significantly (Fig. 2D-F; P $>0.05$ ). In addition, BIO treatment in SW480 and SW620 cells downreg- ulated the expression level of p-GSK-3 $\beta$ (Fig. 2D-F; P<0.05), whereas the effects were slight in total GSK-3 $\beta$ expression (Fig. 2D-F; P $>0.05$ ).

Effect of BIO on apoptosis in SW480 and SW620 cells. After treatment with different concentrations of BIO $(0,1,2$ and $4 \mu \mathrm{M}$ ), apoptosis of SW480 and SW620 cells were examined using flow cytometry (Fig. 3A). When BIO concentration increased, apoptosis of SW480 cells was decreased (Fig. 3B; $\mathrm{P}<0.05$ ), whereas the effects were slight in SW620 cells (Fig. 3C; $\mathrm{P}>0.05)$. After incubation with either $\mathrm{BIO} / 5-\mathrm{Fu} / \mathrm{DDP}$ alone or combined 5-Fu/DDP with BIO, cell apoptosis was examined using flow cytometry (Fig. 3D). Apoptosis was decreased in BIO-treated SW480 cells (Fig. 3E; P<0.05). In addition, BIO treatment significantly decreased apoptosis induced by $5-\mathrm{FU} /$ DDP in SW480 cells (Fig. 3E; P<0.05), whereas the effects were slight in SW620 cells (Fig. 3F; P>0.05).

Effect of BIO on cell cycle progression in SW480 and SW620 cells. Cell cycle distribution was determined by flow cytometry after BIO treatment (Fig. 4A). There was no significant difference in the cell cycle distribution after treatment with $\mathrm{BIO} / 5-\mathrm{Fu}$ alone or combining 5-Fu with $\mathrm{BIO}$ in SW480 and SW620 cells (Fig. 4B; $P>0.05$ ).

In SW480 cells, the percentage of G0/G1 phase cells were decreased, $\mathrm{S}+\mathrm{G} 2 / \mathrm{M}$ phase cells were increased after treatment 

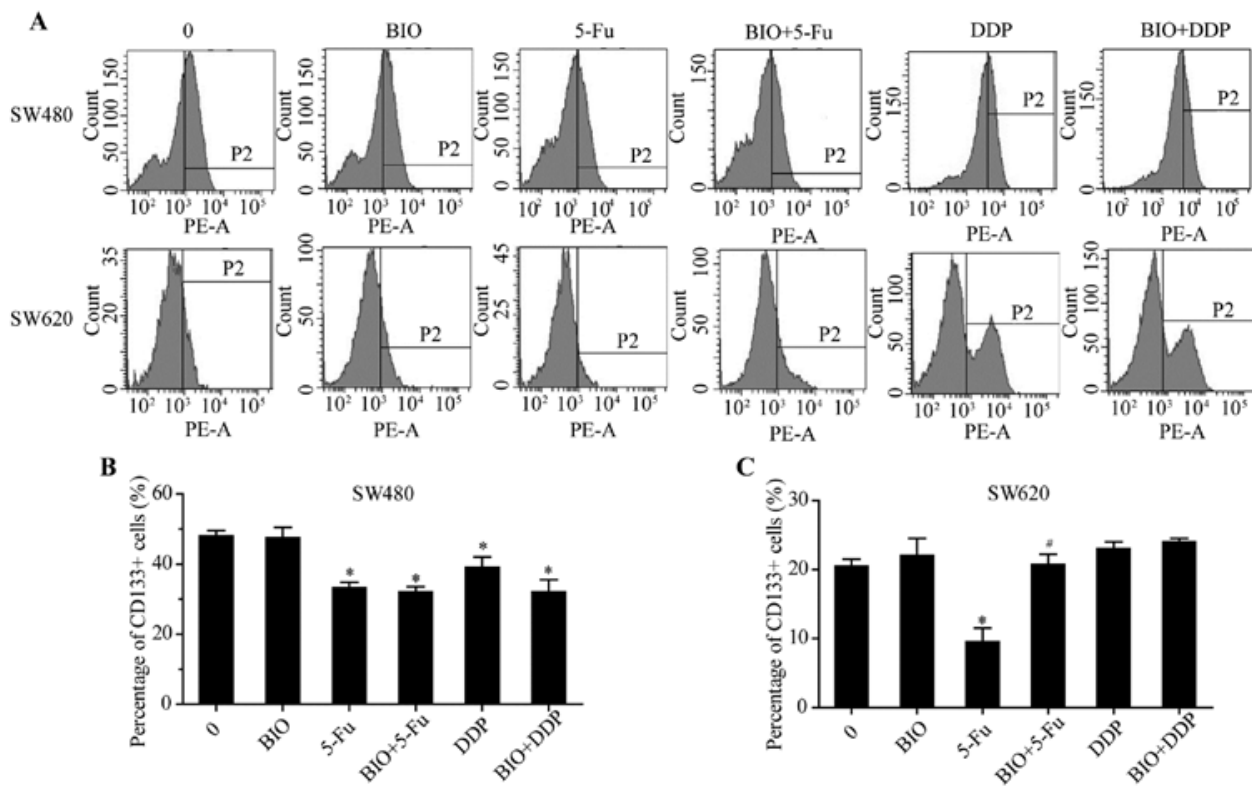

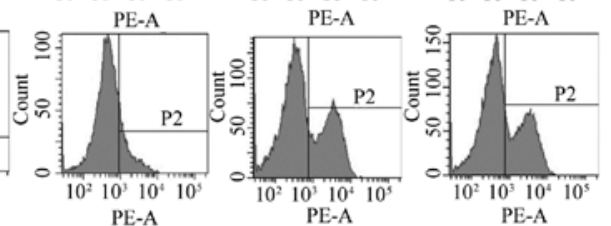

C

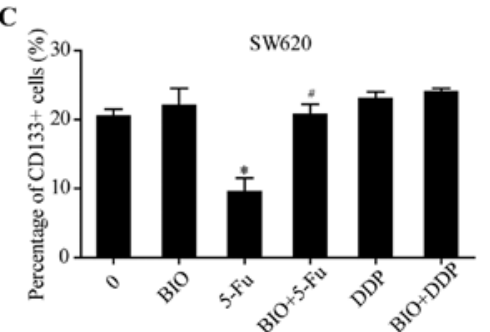

Figure 5. The effects of BIO on the proportion of CD133+ cells of SW480 and SW620 cells. (A) Flow cytometric analysis of the percentage of CD133 ${ }^{+}$cells when treated control/5-FU/DDP alone or combining with BIO $(4 \mu \mathrm{M})$ for $24 \mathrm{~h}$ in SW480 and SW620 cells. (B) Flow cytometric analysis of the percentage of $\mathrm{CD}_{133^{+}}$SW480 cells when treated control/5-FU/DDP alone or combining with BIO. (C) Flow cytometric analysis of the percentage of CD133 ${ }^{+}$SW620 cells when treated control/5-FU/DDP alone or combining with BIO. Data represent mean \pm SD of three independent experiments with technical triplicates for each, and statistical analysis was performed using Student's t-test. ${ }^{*} \mathrm{P}<0.05$ compared with the control. ${ }^{~} \mathrm{P}<0.05$ compared with 5-FU-treated or DDP-treated cells.
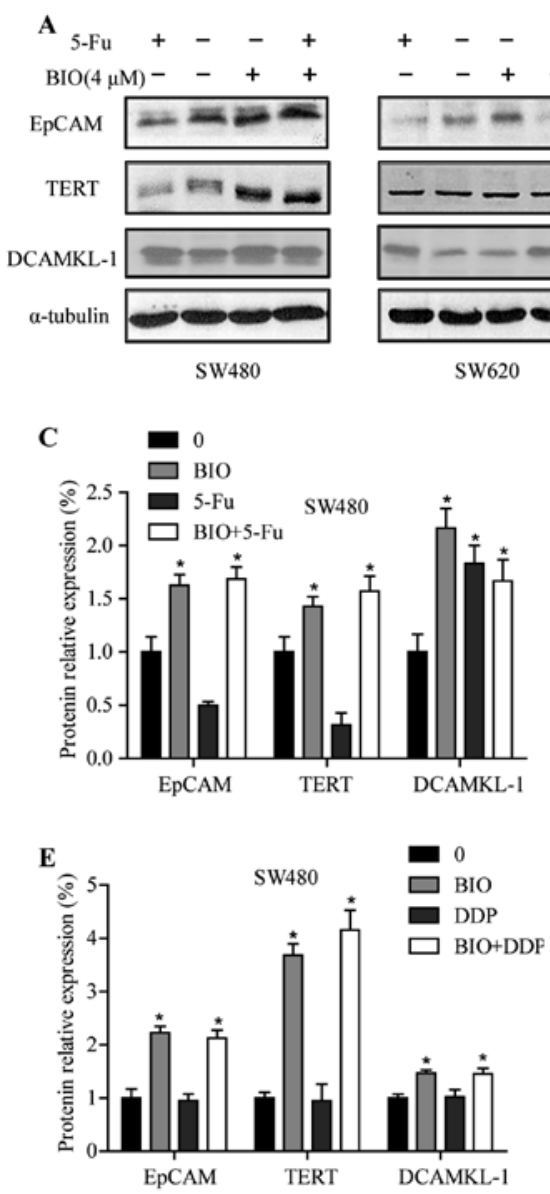

SW620
B

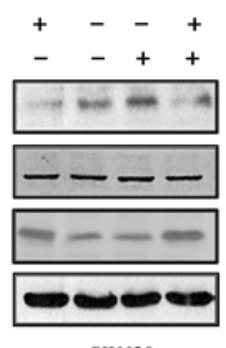

D
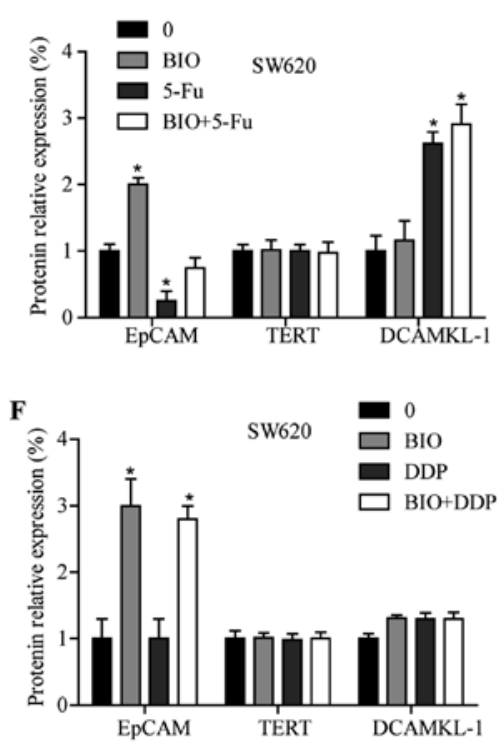

Figure 6. Expression levels of EpCAM, TERT and DCAMKL-1 in SW480 and SW620 cells before and after treatment with BIO, 5-Fu, DDP for 24 h. (A and B) Western blot analyses of EpCAM, TERT and DCAMKL-1 in the indicated SW480/SW620 cell groups. (C and D) Quantitated analysis of EpCAM, TERT and DCAMKL-1 proteins in SW480 and SW620 cells after treated with 5-Fu alone or combining with BIO $(4 \mu \mathrm{M})$ for $24 \mathrm{~h}$. (E and F) Quantitated analysis of EpCAM, TERT and DCAMKL-1 proteins in SW480 and SW620 cells after treated with DDP alone or combined with BIO for $24 \mathrm{~h}$. $\alpha$-tubulin/GAPDH was used as an internal loading control. Values are expressed as protein/ $\alpha$-tubulin or protein/GAPDH. The density of the protein band was assessed using Quantity One software. The data are expressed as means \pm SD of three experiments. ${ }^{*} \mathrm{P}<0.05$ compared with the control. 
A

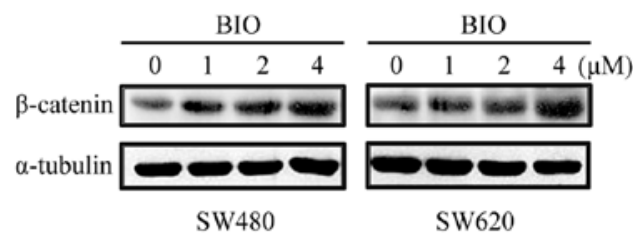

B

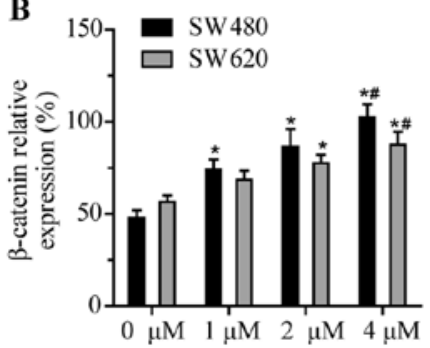

C

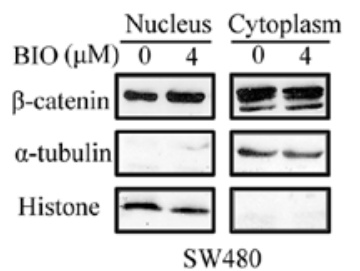

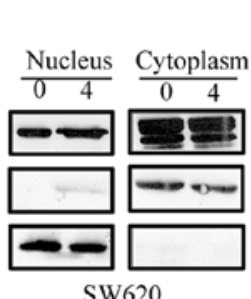

SW620
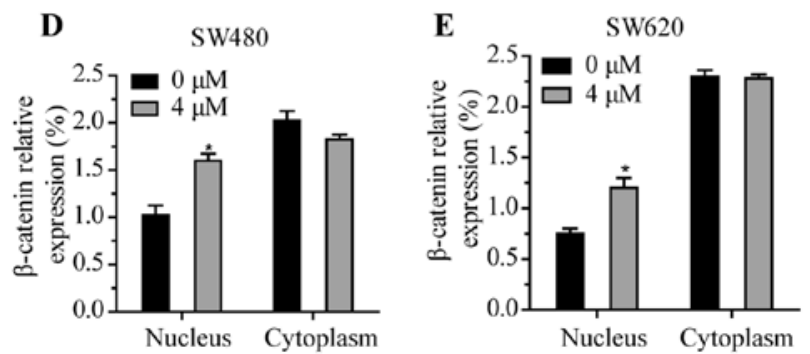

Figure 7. Expression levels of $\beta$-catenin in SW480 and SW620 cells before and after treatment with BIO $(4 \mu \mathrm{M})$ for $24 \mathrm{~h}$. (A and B) Protein expression levels of $\beta$-catenin by western blotting in SW480 and SW620 cells before and after treated with different doses of BIO. $\alpha$-tubulin was used as an internal loading control. Values are expressed as protein/ $\alpha$-tubulin. (C-E) Western blot analysis of $\beta$-catenin proteins in nuclear and cytoplasm after treatment with BIO $(4 \mu \mathrm{M})$ for $24 \mathrm{~h}$. Values are expressed as protein/ $\alpha$-tubulin or protein/histone. The density of the protein band was quantitated using Quantity One software. The data are expressed as mean $\pm \mathrm{SD}$ of three experiments. ${ }^{*} \mathrm{P}<0.05$ compared with the control $(0 \mu \mathrm{M})$ group. ${ }^{*} \mathrm{P}<0.05$ compared with the $1 \mu \mathrm{M}$ group.

with DDP alone or combining DDP with $\mathrm{BIO}$ (Fig. 4B; P<0.05), but no significant difference existed between the two groups (Fig. 4B; P>0.05). In SW620 cells, no significant difference was found in the cell cycle distribution after treatment with DDP alone or combining DDP with BIO (Fig. 4C; P>0.05). Collectively, these results suggest that $\mathrm{BIO}$ has no significant effect on 5-Fu/DDP-induced cell cycle distribution.

Effect of BIO on proportion of CD133+ SW480 and SW620 cells. $\mathrm{CD} 133^{+}$cells were examined using flow cytometry after BIO treatment (Fig. 5A). As shown in Fig. 5B, the proportions of $\mathrm{CD}_{133}{ }^{+} \mathrm{SW} 480$ cells were decreased when either 5 -Fu or DDP was added $(\mathrm{P}<0.05)$. There was no significant difference in the proportions of CD133 ${ }^{+}$SW480 cells between treated with 5-Fu/DDP alone and combining 5-Fu/DDP with BIO (Fig. 5B; P>0.05). In SW620 cells, after treated with 5-FU, the proportions of $\mathrm{CD}_{133^{+}}$cells were decreased (Fig. 5C; $\mathrm{P}<0.05)$. BIO combining 5-Fu treatment reversed the CD133+ cell downregulation trend compared with treatment with $5-\mathrm{Fu}$ alone (Fig. 5C; $\mathrm{P}<0.05$ ). In addition, either treated with DDP alone or combining DDP with BIO has no significantly effect on the proportions of CD133+ SW620 cells (Fig. 5C; P>0.05).

Effect of BIO on CSC marker proteins in SW480 and SW620 cells. To further investigate the effect of BIO on CSC marker protein expression, total protein lysates were prepared and analyzed by western blotting (Fig. 6A and B). As shown in Fig. 6C-F, following treatment of BIO, the expression levels of EpCAM, TERT and DCAMKL-1 were increased in SW480 cells, EpCAM was increased in SW620 cells $(\mathrm{P}<0.05)$, respectively.

In addition, BIO treatment combining 5-Fu/DDP upregulated the expression levels of EpCAM, TERT and DCAMKL-1 in SW480 cells (Fig. 6C and E; P<0.05). In SW620 cells, BIO treatment upregulated the expression levels of DCAMKL-1 in combining with 5-Fu, and upregulated the expression levels of EpCAM in combining with DDP (Fig. 6D and F; P<0.05).

Effect of BIO on $\beta$-catenin expression in SW480 and SW620 cells. To further investigate the effect of $\mathrm{BIO}$ on proteins involved in $\mathrm{WNT} / \beta$-catenin signaling pathway expression, total protein lysates were prepared and analyzed by western blotting. As shown in Fig. 7A, following treated with different doses of BIO, the expression levels of total $\beta$-catenin increased in SW480 and SW620 cells (Fig. 7B; P<0.05), respectively. The effect of BIO on $\beta$-catenin in SW480 and SW620 cells is concentration-dependent. Western blot analysis of $\beta$-catenin protein both nuclear and cytoplasmic (Fig. 7C) displayed that $\beta$-catenin was upregulated in the nuclei of SW480 (Fig. 7D; $\mathrm{P}<0.05)$ and SW620 cells (Fig. 7E; $\mathrm{P}<0.05)$ after treatment with $\mathrm{BIO}$, respectively.

\section{Discussion}

In the present study, to survey the tangible biological behavior alteration induced by $\mathrm{BIO}$ in CRC cells, we conducted cDNA microarrays to pick out the differentially expressed genes after BIO-treatment in SW480 and SW620 cells. The cDNA microarrays identified various genes, including mesenchymal stem cell and cell migration relative genes, significantly aberrant expression in BIO-treated SW480 and SW620 cells. In vitro experimental results indicated that the apoptosis was decreased in BIO-treated SW480 cells. More importantly, the apoptosis induced by $5-\mathrm{Fu} / \mathrm{DDP}$ was also decreased in BIO-treated SW480 cells. The Transwell and boyden chamber experimental results demonstrated that the migration and invasion abilities of SW480 and SW620 cells were decreased after treatment with $\mathrm{BIO}$, accompanying with MMP-9 downregulation and E-cadherin upregulation in these cells. The MMP-9 has been widely documented in many physiological and 


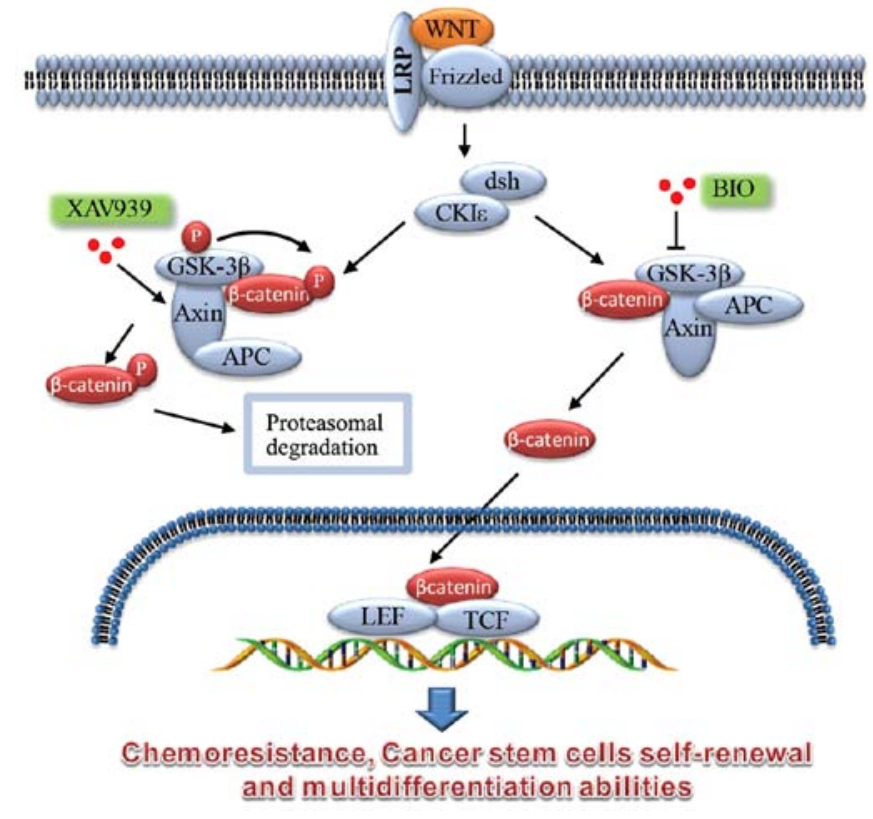

Figure 8 . The proposed model of BIO in WNT signaling pathway. The $\beta$-catenin destruction complex, which consists of Apc, Axin, CKIE and GSK-3 $\beta$, promotes proteasome-mediated proteolysis of phosphorylated $\beta$-catenin. Marked downregulation of the GSK-3 $\beta$ protein activity by BIO, leads to $\beta$-catenin transportation to the nucleus, and then results in adhesive, stemness and chemoresistance increasing in CRC cells.

pathological processes including cell migration, invasion and growth $(25,26)$. E-cadherin, an important adhesive factor, can produce a marked effect on mediating tissue integration and cellular adhesiveness in form of complex with catenin (27). Therefore, cell motility related protein alteration after BIO treatment may result in adhesive activity enhancing the CRC cells.

We found that BIO treatment promotes both adhesive activity and drug resistance in CRC cells, interestingly, in these biological behaviors exist certain correlation in the mechanism. An important theory indicated that cell adhesion mediated drug resistance (CAM-DR) through suppressed drug-induced apoptosis (28). Tumor cells grown as multicellular spheroids were more resistant to chemotherapeutic drugs compared to the same cells grown as dispersed monolayer cell cultures (29). Possible mechanisms have been put forward to account for CAM-DR. E-cadherinmediated intracellular adhesion causing lack of sufficient drug penetration into solid tumors is one of the important molecular mechanisms for CAM-DR (30). In addition, various research has suggested that with the adhesive activity increasing, drug resistance protein such as P-gp was induced to express in cancer cells (31). Our previous research shows that treatment with different concentrations of BIO, the expression level of P-gp and cellular efflux ability were dose-dependently elevated in SW480 and SW620 cells (32). In the present study, our experimental results suggested that BIO treatment promotes adhesive activity through altering the expression levels of E-cadherin and MMP-9 in SW480 and SW620 cells. Therefore, the aberrant expression of E-cadherin and MMP-9 may also contribute to CAM-DR in BIO-treated CRC cells.
Besides adhesiveness promotes drug resistance in BIO-treated cells, we found that the stem cell relative proteins had significant aberrant expression in BIO-treated SW480 and SW620 cells. It is generally known that CSCs are in dormant or slow-growing phase of the cell cycle, such features contributing for their therapeutic refractoriness to chemo/radiation therapy and tumor relapse (33-35). Colorectal CSCs are characterized by different types of CSC markers such as CD133, EpCAM, TERT and DCAMKL-1 (36-39). In the present study, BIO treatment upregulated the expression levels of EpCam, TERT and DCAMKL-1 proteins in SW480 or SW620 cells at various degrees. In addition, treatment with BIO reversed the 5-FU-induced $\mathrm{CD}_{133^{+}}$cell downregulation trend in SW620 cells. These results suggested that the drug resistance ability enhancing after BIO treatment was partly attributed to stemness increasing in CRC cells.

In the present study, we found that the biological behavior alterations of SW480 and SW620 cells after treatment with BIO were not entirely consistent. The reason may be that heterogeneity was present between these two cell lines. Originally, CRC cell line SW480 was isolated from a high-grade primary colon tumor, CRC cell line SW620 was isolated from the same patient's metastatic lymph node at the time of clinical relapse one year later (40). In our previous study, experimental results suggested that heterogeneity of drug resistance was present between metastatic and primary CRC specimens and cell lines (41). We found that the ability of drug resistance in metastatic SW620 cells was greater than primary colorectal cancer cells SW480 owing to cancer stem cells and drug resistance relative protein activation. Therefore, we believe that different reaction after BIO treatment may partly attribute to heterogeneity between SW480 and SW620 cells.

As mentioned above, the effect of BIO on biological behavior of CRC cells was complicated. There are a number of independent studies reporting that BIO could evidently inhibit the activity of GSK- $3 \beta$ through competing with ATP and influencing the phosphorylation of GSK-3 $\beta$ (24). The full activity of GSK-3 $\beta$ generally requires phosphorylation at tyrosine 216, and conversely, phosphorylation at serine 9 inhibits GSK-3 $\beta$ activity (42-44). Recently, the biological effects of small molecule compounds on GSK-3 $\beta$ were widely studied for its potential value on CRC treatment. It is reported that activation of GSK-3 $\beta$ while downregulating the PI3-K/Akt oncogenic pathway by non-steroidal anti-inflammatory drugs (NSAIDs), results uniformly in the chemopreventive and anti-neoplastic effects in the early stage of colon cancer (45). In the present study, treated with BIO in CRC cells, the expression levels of p-GSK-3 $\beta$ (Tyr 216) were decreased both in CRC SW480 and SW620 cells, demonstrating that GSK- $3 \beta$ activity was significantly inhibited by BIO. GSK-3 $\beta$ is the key component of the $\beta$-catenin destruction complex in WNT pathway, and promotes proteasome-mediated proteolysis of phosphorylated $\beta$-catenin (46). As expected, subsequent experimental results showed that BIO treatment upregulation and transportation of $\beta$-catenin to the nucleus of SW480 and SW620 cells. It is generally believed that WNT signaling pathway effector $\beta$-catenin plays an important role in the maintenance of stemness and drug resistance ability of CSCs (47-49), the chemoresistance increasing and stemness enhancing in BIO-treated CRC cells may partly 
attribute to $\beta$-catenin activation. Importantly, GSK-3 $\beta$ is a critical regulatory component of $\mathrm{WNT} / \beta$-catenin, $\mathrm{NF}-\kappa \mathrm{B}$, phosphoinositide 3-kinase (PI3K)/AKT and other signaling pathways (50-53). The present study mainly focused on the effect and mechanism of BIO on WNT/ $\beta$-catenin signaling pathway. However, the important role of other signaling pathways in BIO treated CRC cells, such as NF- $\mathrm{B}$ and PI3K/ AKT, still need to be further evaluated.

Taken together, the results presented here demonstrated that BIO upregulated stemness, adhesive and chemoresistance of CRC cells. In addition, BIO treatment downregulated the activity of GSK-3 $\beta$, upregulated and transported $\beta$-catenin to the nucleus, which may partly result in these biological behavior alterations in CRC cells (Fig. 8). Of note, BIO is a multi-target inhibitor. As proven by various research, BIO inhibits not only GSK-3 $\beta$ activity, but also GSK-3 $\alpha$, JAK1 activities and possbly TYK2 (23-25). In the present study, the cDNA microarray analysis results also suggested that $\mathrm{BIO}$ treatment impact various signaling pathways. Therefore, using more than one inhibitor and specific RNA interference will be a better way to evaluate the role of GSK-3 $\beta$ inhibition in CRC cells for further work. In addition, the activity alterations of these signaling pathways in BIO treated CRC cells were not evaluate in present study. The underlying molecular mechanism causing stemness, adhesive and chemoresistance increase in CRC cells after BIO treatment still need to be further evaluated.

\section{Acknowledgements}

The present study was sponsored by the Guangdong Natural Science Foundation (nos. 2014A030307007 and no. 2017A030307005) and the Sci-Tech Project Foundation of Qingyuan City (no. 2013A009).

\section{References}

1. Torre LA, Bray F, Siegel RL, Ferlay J, Lortet-Tieulent J and Jemal A: Global cancer statistics, 2012. CA Cancer J Clin 65 87-108, 2015

2. Peters GJ, Backus HH, Freemantle S, van Triest B, CodacciPisanelli G, van der Wilt CL, Smid K, Lunec J, Calvert AH, Marsh S, et al: Induction of thymidylate synthase as a 5-fluorouracil resistance mechanism. Biochim Biophys Acta 1587: 194-205, 2002.

3. Guinney J, Dienstmann R, Wang X, de Reyniès A, Schlicker A, Soneson C, Marisa L, Roepman P, Nyamundanda G, Angelino P, et al: The consensus molecular subtypes of colorectal cancer. Nat Med 21: 1350-1356, 2015.

4. Zhang Z, Zhou C, Chang Y, Zhang Z, Hu Y, Zhang F, Lu Y, Zheng L, Zhang W, Li X, et al: Long non-coding RNA CASC11 interacts with hnRNP-K and activates the $\mathrm{WNT} / \beta$-catenin pathway to promote growth and metastasis in colorectal cancer. Cancer Lett 376: 62-73, 2016.

5. Zheng K, Zhou X, Yu J, Li Q, Wang H, Li M, Shao Z, Zhang F, Luo Y, Shen Z, et al: Epigenetic silencing of miR-490-3p promotes development of an aggressive colorectal cancer phenotype through activation of the $\mathrm{Wnt} / \beta$-catenin signaling pathway. Cancer Lett 376: 178-187, 2016.

6. Gao Y, Liu Z, Zhang X, He J, Pan Y, Hao F, Xie L, Li Q, Qiu X and Wang E: Inhibition of cytoplasmic GSK- $3 \beta$ increases cisplatin resistance through activation of $\mathrm{Wnt} / \beta$-catenin signaling in A549/DDP cells. Cancer Lett 336: 231-239, 2013.

7. Vermeulen L, De Sousa E Melo F, van der Heijden M, Cameron K, de Jong JH, Borovski T, Tuynman JB, Todaro M, Merz $\mathrm{C}$, Rodermond $\mathrm{H}$, et al: Wnt activity defines colon cancer stem cells and is regulated by the microenvironment. Nat Cell Biol 12: 468-476, 2010.
8. Morin PJ, Sparks AB, Korinek V, Barker N, Clevers H, Vogelstein B and Kinzler KW: Activation of beta-catenin-Tcf signaling in colon cancer by mutations in beta-catenin or APC. Science 275: 1787-1790, 1997.

9. Kimelman D and $\mathrm{Xu}$ W: beta-catenin destruction complex: Insights and questions from a structural perspective. Oncogene 25: 7482-7491, 2006

10. Prunier C, Hocevar BA and Howe PH: Wnt signaling: Physiology and pathology. Growth Factors 22: 141-150, 2004.

11. Cui J, Jiang W, Wang S, Wang L and Xie K: Role of Wnt/ $\beta$-catenin signaling in drug resistance of pancreatic cancer. Curr Pharm Des 18: 2464-2471, 2012.

12. Wu X, Luo F, Li J, Zhong X and Liu K: Tankyrase 1 inhibitior XAV939 increases chemosensitivity in colon cancer cell lines via inhibition of the Wnt signaling pathway. Int J Oncol 48: 1333-1340, 2016.

13. Woodgett JR: Molecular cloning and expression of glycogen synthase kinase-3/factor A. EMBO J 9: 2431-2438, 1990.

14. Embi N, Rylatt DB and Cohen P: Glycogen synthase kinase-3 from rabbit skeletal muscle. Separation from cyclic-AMPdependent protein kinase and phosphorylase kinase. Eur J Biochem 107: 519-527, 1980.

15. Lucas JJ, Hernández F, Gómez-Ramos P, Morán MA, Hen R and Avila J: Decreased nuclear beta-catenin, tau hyperphosphorylation and neurodegeneration in GSK-3beta conditional transgenic mice. EMBO J 20: 27-39, 2001.

16. Takahashi-Yanaga $\mathrm{F}$ and Sasaguri T: GSK-3beta regulates cyclin D1 expression: A new target for chemotherapy. Cell Signal 20: 581-589, 2008.

17. Grassilli E, Narloch R, Federzoni E, Ianzano L, Pisano F, Giovannoni R, Romano G, Masiero L, Leone BE, Bonin S, et al: Inhibition of GSK3B bypass drug resistance of p53-null colon carcinomas by enabling necroptosis in response to chemotherapy. Clin Cancer Res 19: 3820-3831, 2013.

18. Wang Z, Smith KS, Murphy M, Piloto O, Somervaille TC and Cleary ML: Glycogen synthase kinase 3 in MLL leukaemia maintenance and targeted therapy. Nature 455: 1205-1209, 2008.

19. Forde JE and Dale TC: Glycogen synthase kinase 3: A key regulator of cellular fate. Cell Mol Life Sci 64: 1930-1944, 2007.

20. Sato N, Meijer L, Skaltsounis L, Greengard P and Brivanlou AH: Maintenance of pluripotency in human and mouse embryonic stem cells through activation of Wnt signaling by a pharmacological GSK-3-specific inhibitor. Nat Med 10: 55-63, 2004.

21. Liu L, Nam S, Tian Y, Yang F, Wu J, Wang Y, Scuto A, Polychronopoulos P, Magiatis P, Skaltsounis L, et al: 6-Bromoindirubin-3'-oxime inhibits JAK/STAT3 signaling and induces apoptosis of human melanoma cells. Cancer Res 71: 3972-3979, 2011.

22. Yu AS and Zhao L: Effects of the GSK-3 $\beta$ inhibitor (2Z,3E)6-bromoindirubin-3'-oxime upon ovarian cancer cells. Tumour Biol 37: 4857-4864, 2016.

23. Eslaminejad MB, Salami F, Mehranjani MS, Abnoosi M and Eftekhari-yazdi P: BIO treatment enhances rat marrow-derived mesenchymal stem cell in vitro proliferation and viability. Physiol Pharmacol 13: 121-126, 2009.

24. Tseng AS, Engel FB and Keating MT: The GSK-3 inhibitor BIO promotes proliferation in mammalian cardiomyocytes. Chem Biol 13: 957-963, 2006.

25. Lu L, Zhang Q, Wu K, Chen X, Zheng Y, Zhu C and Wu J: Hepatitis C virus NS3 protein enhances cancer cell invasion by activating matrix metalloproteinase- 9 and cyclooxygenase- 2 through ERK/p38/NF- $\mathrm{BB}$ signal cascade. Cancer Lett 356: 470-478, 2015.

26. Che YL, Luo SJ, Li G, Cheng M, Gao YM, Li XM, Dai JM, He H, Wang J, Peng HJ, et al: The C3G/Rap1 pathway promotes secretion of MMP-2 and MMP-9 and is involved in serous ovarian cancer metastasis. Cancer Lett 359: 241-249, 2015.

27. Batlle E, Sancho E, Francí C, Domínguez D, Monfar M, Baulida J and García De Herreros A: The transcription factor snail is a repressor of E-cadherin gene expression in epithelial tumour cells. Nat Cell Biol 2: 84-89, 2000.

28. Zhou BP, Deng J, Xia W, Xu J, Li YM, Gunduz M and Hung MC: Dual regulation of Snail by GSK-3beta-mediated phosphorylation in control of epithelial-mesenchymal transition. Nat Cell Biol 6: 931-940, 2004.

29. Petitclerc E, Deschesnes RG, Côté MF, Marquis C, Janvier R, Lacroix J, Miot-Noirault E, Legault J, Mounetou E, Madelmont JC, et al: Antiangiogenic and antitumoral activity of phenyl-3-(2-chloroethyl)ureas: A class of soft alkylating agents disrupting microtubules that are unaffected by cell adhesionmediated drug resistance. Cancer Res 64: 4654-4663, 2004. 
30. Shain KH and Dalton WS: Cell adhesion is a key determinant in de novo multidrug resistance (MDR): New targets for the prevention of acquired MDR. Mol Cancer Ther 1: 69-78, 2001.

31. Damiano JS, Cress AE, Hazlehurst LA, Shtil AA and Dalton WS Cell adhesion mediated drug resistance (CAM-DR): Role of integrins and resistance to apoptosis in human myeloma cell lines. Blood 93: 1658-1667, 1999.

32. Liu KP, Luo F, Xie SM, Tang LJ, Chen MX, Wu XF, Zhong XY and Zhao T: Glycogen synthase kinase $3 \beta$ inhibitor $\left(2{ }^{\prime} Z, 3^{\prime} E\right)$ 6-Bromo-indirubin-3'-oxime enhances drug resistance to 5 -fluorouracil chemotherapy in colon cancer cells. Chin J Cancer Res 24: 116-123, 2012.

33. Yang HZ, Ma Y, Zhou Y, Xu LM, Chen XJ, Ding WB and Zou HB: Autophagy contributes to the enrichment and survival of colorectal cancer stem cells under oxaliplatin treatment. Cancer Lett 361: 128-136, 2015.

34. De Angelis ML, Zeuner A, Policicchio E, Russo G, Bruselles A, Signore M, Vitale S, De Luca G, Pilozzi E, Boe A, et al: Cancer stem cell-based models of colorectal cancer reveal molecular determinants of therapy resistance. Stem Cells Transl Med 5: 511-523, 2016.

35. McCubrey JA, Steelman LS, Abrams SL, Misaghian N, Chappell WH, Basecke J, Nicoletti F, Libra M, Ligresti G, Stivala F, et al: Targeting the cancer initiating cell: The ultimate target for cancer therapy. Curr Pharm Des 18: 1784-1795, 2012.

36. Nakanishi Y, Seno H, Fukuoka A, Ueo T, Yamaga Y, Maruno T, Nakanishi N, Kanda K, Komekado H, Kawada M, et al: Dclk1 distinguishes between tumor and normal stem cells in the intestine. Nat Genet 45: 98-103, 2013.

37. Imrich S, Hachmeister M and Gires O: EpCAM and its potential role in tumor-initiating cells. Cell Adhes Migr 6: 30-38, 2012.

38. Merlos-Suárez A, Barriga FM, Jung P, Iglesias M, Céspedes MV, Rossell D, Sevillano M, Hernando-Momblona X, da Silva-Diz V, Muñoz P, et al: The intestinal stem cell signature identifies colorectal cancer stem cells and predicts disease relapse. Cell Stem Cell 8: 511-524, 2011

39. Elsaba TM, Martinez-Pomares L, Robins AR, Crook S, Seth R, Jackson D, McCart A, Silver AR, Tomlinson IP and Ilyas M: The stem cell marker CD133 associates with enhanced colony formation and cell motility in colorectal cancer. PLoS One 5: e10714, 2010.

40. Leibovitz A, Stinson JC, McCombs WB III, McCoy CE, Mazur KC and Mabry ND: Classification of human colorectal adenocarcinoma cell lines. Cancer Res 36: 4562-4569, 1976.
41. Luo F, Li J, Wu S, Wu X, Chen M, Zhong X and Liu K: Comparative profiling between primary colorectal carcinomas and metastases identifies heterogeneity on drug resistance. Oncotarget 7: 63937-63949, 2016.

42. Jiang H, Guo W, Liang X and Rao Y: Both the establishment and the maintenance of neuronal polarity require active mechanisms: Critical roles of GSK-3beta and its upstream regulators. Cell 120: 123-135, 2005.

43. Doble BW and Woodgett JR: GSK-3: Tricks of the trade for a multi-tasking kinase. J Cell Sci 116: 1175-1186, 2003.

44. Lin CL, Wang JY, Huang YT, Kuo YH, Surendran K and Wang FS: Wnt/beta-catenin signaling modulates survival of high glucose-stressed mesangial cells. J Am Soc Nephrol 17: 2812-2820, 2006.

45. Jain S, Ghanghas P, Rana $C$ and Sanyal SN: Role of GSK-3 $\beta$ in regulation of canonical Wnt/ $\beta$-catenin signaling and PI3-K/Akt oncogenic pathway in colon cancer. Cancer Invest 35: 473-483, 2017.

46. Ikeda S, Kishida S, Yamamoto H, Murai H, Koyama S and Kikuchi A: Axin, a negative regulator of the Wnt signaling pathway, forms a complex with GSK-3beta and beta-catenin and promotes GSK-3beta-dependent phosphorylation of beta-catenin. EMBO J 17: 1371-1384, 1998.

47. Wang WJ, Wu MY, Shen M, Zhi Q, Liu ZY, Gong FR, Tao M and $\mathrm{Li} \mathrm{W}$ : Cantharidin and norcantharidin impair stemness of pancreatic cancer cells by repressing the $\beta$-catenin pathway and strengthen the cytotoxicity of gemcitabine and erlotinib. Int J Oncol 47: 1912-1922, 2015

48. Reya T, Morrison SJ, Clarke MF and Weissman IL: Stem cells, cancer, and cancer stem cells. Nature 414: 105-111, 2001.

49. Todaro M, Francipane MG, Medema JP and Stassi G: Colon cancer stem cells: Promise of targeted therapy. Gastroenterology 138: 2151-2162, 2010.

50. Clevers $\mathrm{H}$ : Wnt/beta-catenin signaling in development and disease. Cell 127: 469-480, 2006.

51. Cantley LC: The phosphoinositide 3-kinase pathway. Science 296: 1655-1657, 2002.

52. Hennessy BT, Smith DL, Ram PT, Lu Y and Mills GB: Exploiting the PI3K/AKT pathway for cancer drug discovery. Nat Rev Drug Discov 4: 988-1004, 2005

53. Buss H, Dörrie A, Schmitz ML, Frank R, Livingstone M, Resch K and Kracht M: Phosphorylation of serine 468 by GSK-3beta negatively regulates basal p65 NF-kappaB activity. J Biol Chem 279: 49571-49574, 2004. 